\title{
Quantification of Inflammation Within Rabbit Atherosclerotic Plaques Using the Macrophage-Specific CT Contrast Agent N1177: A Comparison with ${ }^{18}$ F-FDG PET/CT and Histology
}

Fabien Hyafil ${ }^{1,2}$, Jean-Christophe Cornily ${ }^{1,3}$, James H.F. Rudd ${ }^{1,4}$, Josef Machac ${ }^{5}$, Laurent J. Feldman ${ }^{2}$, and Zahi A. Fayad ${ }^{1}$

${ }^{1}$ Translational and Molecular Imaging Institute and Imaging Science Laboratories, Mount Sinai School of Medicine, New York, New York; ${ }^{2}$ Department of Cardiology and Nuclear Medicine, Bichat Hospital, Assistance Publique-Hôpitaux de Paris and Institut National de la Santé et de la Recherche Médicale, 698, Paris, France; ${ }^{3}$ Department of Cardiology, Brest University Hospital, Brest, France, and ORPHY Laboratory, Université de Brest, Brest, France; ${ }^{4}$ Division of Cardiovascular Medicine, University of Cambridge, Cambridge, United Kingdom; and ${ }^{5}$ Department of Nuclear Medicine, Mount Sinai Hospital, New York, New York

Macrophages play a key role in atherosclerotic plaque rupture. The iodine-based contrast agent N1177 accumulates in macrophages, allowing for their detection with CT. In this study, we tested whether the intensity of enhancement detected with CT in the aortic wall of rabbits injected with N1177 correlated with inflammatory activity evaluated with ${ }^{18} \mathrm{~F}-\mathrm{FDG}$ PET/CT and macrophage density on histology. Methods: Atherosclerotic plaques were induced in the aorta of New Zealand White rabbits $(n=7)$ by a repeated balloon injury ( $4 \mathrm{wk}$ apart) and 4 mo of hyperlipemic diet. Noninjured rabbits, fed a chow diet, were used as controls $(n=3)$. A CT scan of the aorta $(n=10)$ was acquired in each rabbit before, during, and at $2 \mathrm{~h}$ after intravenous injection of $\mathrm{N} 1177$ (250 $\mathrm{mg}$ of iodine/ $\mathrm{kg}$ ). One week later, the same rabbits underwent PET/CT $3 \mathrm{~h}$ after injection of ${ }^{18} \mathrm{~F}-\mathrm{FDG}$ (37 MBq/kg [1 $\mathrm{mCi} / \mathrm{kg}]$ ). CT enhancement was calculated as the difference in aortic wall densities between images obtained before and images obtained at $2 \mathrm{~h}$ after injection of N1177. Mean standardized uptake values were measured on PET axial slices of the aorta in regions of interest encompassing the vessel wall. Macrophage density was measured by immunohistology (anti-RAM-11 antibody) on corresponding aortic cross-sections. Results: N1177-enhanced CT measured stronger enhancement in the aortic wall of atherosclerotic rabbits than in control rabbits (10.0 \pm 5.2 vs. $2.0 \pm 2.1$ Hounsfield units, respectively; $P<$ $0.05)$. After the injection of ${ }^{18} \mathrm{~F}-\mathrm{FDG}, \mathrm{PET}$ detected higher standardized uptake values in the aortic wall of atherosclerotic rabbits than in control rabbits $(0.61 \pm 0.12$ vs. $0.21 \pm 0.02 ; P<$ 0.05). The intensity of enhancement in the aortic wall measured with CT after injection of $\mathrm{N} 1177$ correlated with ${ }^{18} \mathrm{~F}-\mathrm{FDG}$ uptake on PET/CT ( $r=0.61, P<0.001)$ and macrophage density on immunohistology ( $r=0.63, P<0.001)$. Conclusion: The inten-

Received Dec. 2, 2008; revision accepted Feb. 27, 2009.

For correspondence or reprints contact: Zahi A. Fayad, Mount Sinai School of Medicine, Translational and Molecular Imaging Institute, One

Gustave L. Levy Place, Box 1234, New York, NY 10029.

E-mail: zahi.fayad@mssm.edu

COPYRIGHT $\odot 2009$ by the Society of Nuclear Medicine, Inc. sity of enhancement detected with CT in the aortic wall of rabbits injected with $\mathrm{N} 1177$ correlates with intense uptake of ${ }^{18} \mathrm{~F}-\mathrm{FDG}$ measured with PET and with macrophage density on histology, suggesting a role for $\mathrm{N} 1177$ in noninvasive identification of high-risk atherosclerotic plaques with CT.

Key Words: atherosclerosis; computed tomography; contrast agents; macrophages; positron emission tomography

J Nucl Med 2009; 50:959-965

DOI: 10.2967/jnumed.108.060749

\section{A} cute coronary syndromes in about two thirds of patients are caused by the disruption of the fibrous cap in so-called high-risk or vulnerable atherosclerotic plaques (1). Sudden exposure of the underlying necrotic core to blood triggers the formation of a thrombus, which can occlude the coronary artery, leading to myocardial necrosis (2). Highrisk atherosclerotic plaques are characterized by their specific cellular and biologic composition rather than by their impact on the vessel lumen (3). Common characteristics of high-risk plaques (4) are a large necrotic core, an overlying fibrous cap measuring less than $65 \mu \mathrm{m}$ thick, strong macrophage infiltration, and positive remodeling (i.e., development of the plaque toward the external part of the artery). Macrophages play a key role in acute plaque destabilization and thrombus formation; they secrete proteases that digest the extracellular matrix and weaken the protective fibrous cap covering the atheromatous core and release in atherosclerotic plaques large amounts of tissue factor that accelerates thrombus formation after plaque rupture (5). Moreover, macrophage density measured by immunohistology was found to be higher in atherosclerotic 
plaques obtained from patients with recent acute coronary syndromes than in plaques obtained from patients with stable cardiovascular disease (6). Therefore, detection of high-risk plaques using noninvasive imaging could help to identify patients susceptible to plaque rupture and reduce the rate of acute coronary syndromes by early implementation of therapies aimed at plaque stabilization (7).

PET with ${ }^{18} \mathrm{~F}-\mathrm{FDG}$ is currently one of the most promising imaging modalities for the detection of inflammatory cells in atherosclerotic plaques $(8) .{ }^{18} \mathrm{~F}-\mathrm{FDG}$ competes with glucose for uptake into metabolically active cells, including macrophages in atherosclerotic plaques, and becomes trapped inside cells after phosphorylation. Accumulation of ${ }^{18} \mathrm{~F}-\mathrm{FDG}$ in carotid atherosclerotic plaques strongly correlates with the intensity of macrophage infiltration on histology (9). Similar relationships between ${ }^{18}$ F-FDG signal and histology have also been found in the rabbit model of atherosclerosis (10-12). In addition, symptomatic patients (i.e., those patients with a recent ischemic cerebral event), compared with asymptomatic patients, showed a significantly higher ${ }^{18} \mathrm{~F}-\mathrm{FDG}$ uptake in carotid plaques (13). However, evaluation of coronary atherosclerotic plaques with ${ }^{18} \mathrm{~F}$-FDG is hampered by the low spatial resolution of PET, cardiac and respiratory motion, and intense ${ }^{18} \mathrm{~F}-\mathrm{FDG}$ uptake by normal myocardium in about half of the patients (14).

In contrast, multidetector CT (MDCT) offers high spatial and temporal resolution, allowing the detection of atherosclerotic plaques in coronary arteries. Some major characteristics of high-risk plaques, such as large volume (15), positive remodeling (16), and absence of or moderate calcifications, can be identified in the coronary arteries using MDCT combined with the intravenous injection of a bolus of small iodinated molecules. However, densities and volumes of plaques measured with MDCT are hampered by a low reproducibility of the measurements related to, among other factors, the strong influence and high variability of nearby luminal enhancement $(17,18)$. In addition, MDCT cannot evaluate the presence or activity of inflammatory cells in atherosclerotic plaques.

In a recently published study (19), we described the CT contrast agent N1177, composed of iodinated nanoparticles dispersed with surfactant. We showed that N1177 given intravenously to rabbits accumulates in macrophages within atherosclerotic plaques $2 \mathrm{~h}$ after injection, allowing for identification of these cells with CT. In this study, we tested whether the intensity of enhancement detected with $\mathrm{CT}$ in the aortic wall of atherosclerotic rabbits $2 \mathrm{~h}$ after injection of N1177 correlated with inflammatory activity evaluated with ${ }^{18}$ F-FDG PET and macrophage density measured on immunohistology of corresponding sections.

\section{MATERIALS AND METHODS}

\section{Animal Model}

Aortic atherosclerotic plaques were induced in male New Zealand White rabbits ( $n=7$; mean age, 4 mo; mean weight, $3.1 \pm 0.2 \mathrm{~kg}$ ) (Covance) by a combination of 4 mo of a high-cholesterol diet
(4.7\% palm oil and $0.3 \%$ cholesterol-enriched diet; Research Diet Inc.) and repeated balloon injury of the aorta ( 2 and $6 \mathrm{wk}$ after the start of the high-cholesterol diet). Aortic injury was performed from the aortic arch to the iliac bifurcation with a 4-French Fogarty embolectomy catheter (Edwards Lifesciences) introduced through the femoral artery. All procedures were performed under general anesthesia induced by an intramuscular injection of ketamine (20 $\mathrm{mg} / \mathrm{kg}$; Fort Dodge Animal Health) and xylazine $(10 \mathrm{mg} / \mathrm{kg}$; Bayer Corp.). Noninjured New Zealand White rabbits, fed a normal chow diet, were used as controls $(n=3)$. All experiments were approved by the Mount Sinai School of Medicine Institute Animal Care and Use Committee.

\section{CT Protocol}

After anesthesia induced by an intramuscular injection of ketamine $(20 \mathrm{mg} / \mathrm{kg})$, xylazine $(10 \mathrm{mg} / \mathrm{kg})$, and acepromazine $(0.5 \mathrm{mg} / \mathrm{kg})$ and placement of a 22-gauge catheter in the marginal ear vein, rabbits were imaged before, during, and at $2 \mathrm{~h}$ (as optimized previously in a study by Hyafil et al. (19)) after the intravenous injection of N1177 (generously provided by Nanoscan Imaging), at a dose equivalent to $250 \mathrm{mg}$ of iodine per kilogram of body weight. Images were acquired using a 64-slice multidetector CT scanner (Sensation 64; Siemens Medical Solutions). Parameters for CT acquisitions were adapted from a clinical protocol used for coronary imaging (except for electrocardiogram gating and current intensity) as follows: tube voltage, $120 \mathrm{kV}$; current intensity, $180 \mathrm{~mA}$; rotation time, $330 \mathrm{~ms}$; detector collimation, $32 \times 0.6 \mathrm{~mm}$; and table feed, $15 \mathrm{~mm} /$ rotation. In-plane resolution of this CT protocol was estimated at $0.4 \times 0.4 \mathrm{~mm}$. Axial slices ( $0.4 \mathrm{~mm}$ thick) were reconstructed on the scanner with a $30 \mathrm{f}$ medium-smooth kernel. The field of view was $100 \times 100 \mathrm{~mm}$, and the data were acquired in a $512 \times 512$ data matrix, giving a pixel size of $0.2 \times 0.2 \mathrm{~mm}$.

\section{PET}

One week after CT, the same rabbits were imaged using a combined PET/CT scanner (Discovery LS; GE Healthcare). PET images were acquired for $10 \mathrm{~min}, 3 \mathrm{~h}$ after intravenous injection of ${ }^{18}$ F-FDG (37 MBq [1 mCi/kg]) (to lower blood ${ }^{18}$ F-FDG activity). Because preliminary studies and published data (11) demonstrated better aorta image quality with 3 -dimensional versus 2 -dimensional acquisition, images were acquired in 3-dimensional mode. The acquisition covered the area from the diaphragm to the aortic bifurcation. The field of view was $15 \times 15 \mathrm{~cm}$, with a $256 \times 256$ data matrix, giving an in-plane spatial resolution of $5 \times 5 \mathrm{~mm}$. Threedimensional reconstructions were performed with a $512 \times 512 \times$ 512 data matrix, giving a final voxel size of $2.5 \times 2.5 \times 2.5 \mathrm{~mm}$. In addition to a PET scan, a non-contrast-enhanced CT scan was obtained in each rabbit using the 16-slice PET/CT machine. Axial slices $(0.4 \mathrm{~mm}$ thick) were reconstructed on the scanner with a $30 \mathrm{f}$ medium-smooth kernel. The field of view was $100 \times 100 \mathrm{~mm}$, and the data were acquired in a $512 \times 512$ data matrix, giving a pixel size of $0.2 \times 0.2 \mathrm{~mm}$.

\section{Image Analysis}

For CT image analysis, densities of the aortic wall were evaluated every $5 \mathrm{~mm}$ on axial CT slices of the abdominal aorta of rabbits $(n=10)$. A series of axial slices $(n=18)$ was matched, using the vertebrae as landmarks. After delineation of the aortic lumen, 12 circular regions of interest (ROIs) of 12 pixels each, adjacent to the lumen, were placed on the CT scan acquired during the injection of contrast agent. Densities were measured in the 
same ROIs in the CT scans acquired before and at $2 \mathrm{~h}$ after the injection of each contrast agent, using a dedicated workstation (Leonardo; Siemens Medical Solutions). Results were expressed in Hounsfield units (HUs) as the average enhancement in each slice of abdominal aortic segment. For comparison with PET/CT, abdominal aortas were divided into proximal, mid, and distal regions of equal length $(30 \mathrm{~mm})$. All CT measurements were performed by an independent operator unaware of the results of PET and immunohistology.

For PET analysis, acquisition data were reconstructed using the Fourier rebinning-iterative algorithm and displayed in 3 orthogonal planes. Average standardized uptake values (SUVs) were recorded on contiguous 5-mm-thick axial slices of aorta $(n=18)$ from circular ROIs encompassing the vessel wall. No corrections were made for blood signal because previous work (11) had demonstrated that blood activity was minimal at the imaging time point ( $3 \mathrm{~h}$ after ${ }^{18} \mathrm{~F}$-FDG injection). Results were expressed as the average SUV in each aortic region. All PET measurements were performed by an independent operator unaware of the results of both CT and immunohistology.

To study the correlations between the results obtained with N1177-enhanced CT and PET, the scans were matched using the vertebrae as landmarks. PET SUVs were measured in the corresponding 30-mm-long aortic segments analyzed by N1177enhanced CT.

\section{Immunohistology}

After PET, rabbits were euthanized by an intravenous injection of sodium pentobarbital $(120 \mathrm{mg} / \mathrm{kg}$ ) (Sleepaway; Fort Dodge Animal Health). A bolus of heparin was injected before euthanasia to prevent clot formation. Aortas were excised, fixed for $24 \mathrm{~h}$ in $4 \%$ paraformaldehyde, and embedded in paraffin. Slices $(5 \mu \mathrm{m}$ thick) were sectioned and matched to CT axial slices as previously described (20). Macrophages were detected on adjacent slices by immunohistochemistry using a monoclonal mouse antibody directed against RAM-11, a marker of rabbit macrophage cytoplasm (dilution, 1:50) (Dako), as previously described (20). The luminal area and the area bounded by the internal elastic laminae were measured on each arterial cross-section using the Image-Pro Plus software (Media Cybernetics) and served to compute intimal area. The total macrophage-rich (RAM-11-positive) area was measured digitally using an automated, contrast-based, area analysis function of the Image-Pro Plus software. Macrophage density was calculated as the ratio between macrophage and intimal areas.

\section{Statistical Analysis}

Numeric values are expressed as mean \pm SE. Values of $P$ less than 0.05 were considered significant. Statistical analysis was conducted with SPSS software (SPSS Inc.). The enhancement detected with N1177 CT and the SUV measured with ${ }^{18} \mathrm{~F}-\mathrm{FDG}$ PET in atherosclerotic and control rabbits were compared using a 2-tailed Student $t$ test. Correlations were performed using Pearson testing.

\section{RESULTS}

\section{Atherosclerotic Versus Control Rabbits}

Two hours after injection of N1177, the density of the aortic wall (Fig. 1) measured with CT was higher in atherosclerotic rabbits than in control rabbits, compared with preinjection values $(31.5 \pm 3.5$ vs. $21.5 \pm 1.8 \mathrm{HUs}$ and $20.4 \pm 1.5$ vs. $18.4 \pm 0.5 \mathrm{HUs}$, respectively). In addition, N1177-enhanced CT measured stronger enhancement in the aortic wall of atherosclerotic rabbits than in control rabbits $(10.0 \pm 5.2$ vs. $2.0 \pm 2.1 \mathrm{HUs}$, respectively; $P<0.05)$. Similarly, at $3 \mathrm{~h}$ after the injection of ${ }^{18} \mathrm{~F}-\mathrm{FDG}$, PET detected higher SUVs in the aortic wall of atherosclerotic rabbits than in control rabbits $(0.61 \pm 0.12$ vs. $0.21 \pm$ 0.02 , respectively; $P<0.05)$.

\section{Correlation Between N1177-Enhanced CT and ${ }^{18}$ F-FDG PET}

In the same rabbit, detection of strong enhancement in the aortic wall at $2 \mathrm{~h}$ after the injection of N1177 with CT was associated with areas of high activity $3 \mathrm{~h}$ after the injection of ${ }^{18}$ F-FDG with PET (Fig. 2). We found a good correlation (Fig. 3) between the intensity of enhancement in the aortic wall detected with N1177-enhanced CT and SUV measured with PET at $3 \mathrm{~h}$ after the injection of ${ }^{18} \mathrm{~F}-\mathrm{FDG}$ $(r=0.61, P<0.001)$.

\section{Correlation Between N1177-Enhanced CT and Macrophage Density}

Atherosclerotic plaques showing a strong enhancement with CT 2 h after injection of N1177 contained an intense macrophage infiltration on corresponding sections by immunohistology (Fig. 4). In addition, we found a good correlation $(r=0.63)$ between the intensity of enhancement measured in atherosclerotic plaques with N1177-enhanced $\mathrm{CT}$ and macrophage density evaluated on corresponding axial cross-sections by immunohistology (Fig. 5A). Both intensities of enhancement with N1177 CT and SUV measured with ${ }^{18}$ F-FDG PET demonstrated good correlations, with

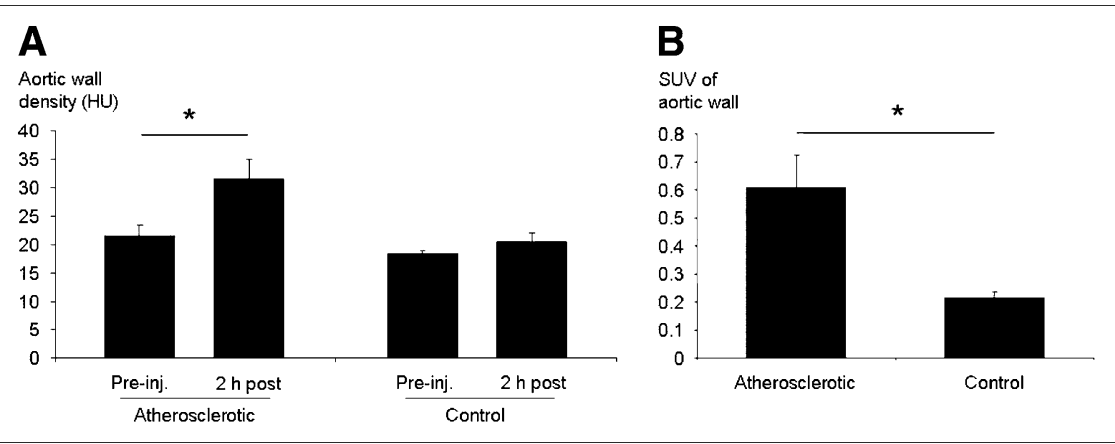

FIGURE 1. Comparison of N1177enhanced CT and ${ }^{18} \mathrm{~F}$-FDG PET in atherosclerotic vs. control rabbits. (A) Stronger enhancement was measured with CT in aortic wall of atherosclerotic rabbits than in control rabbits at $2 \mathrm{~h}$ after injection of N1177. (B) Similarly, higher SUVs were detected with PET in aortic wall of atherosclerotic rabbits than in control rabbits at $3 \mathrm{~h}$ after injection of ${ }^{18} \mathrm{~F}-\mathrm{FDG}$. ${ }^{\star} P<0.05$. inj. $=$ injection. 
FIGURE 2. N1177-enhanced CT and corresponding ${ }^{18} \mathrm{~F}-\mathrm{FDG}$ PET of aorta from atherosclerotic rabbit. Fused PET/ CT coronal view of aorta obtained at $3 \mathrm{~h}$ after injection of ${ }^{18} \mathrm{~F}-\mathrm{FDG}(\mathrm{A})$ and corresponding axial aortic sections acquired before ( $B$ and $D)$ and at $2 \mathrm{~h}$ after rabbit, aortic regions with high $(A$; red cross) and low (A; blue cross) activities identified with PET at $3 \mathrm{~h}$ after injection of ${ }^{18} \mathrm{~F}-\mathrm{FDG}$ were associated with strong (E; red cross) and weak (C; blue cross) intensities of enhancement detected with CT at $2 \mathrm{~h}$ after injection of N1177 on corresponding axial views, respectively. injection of $\mathrm{N} 1177$ (C and $\mathrm{E})$. In same
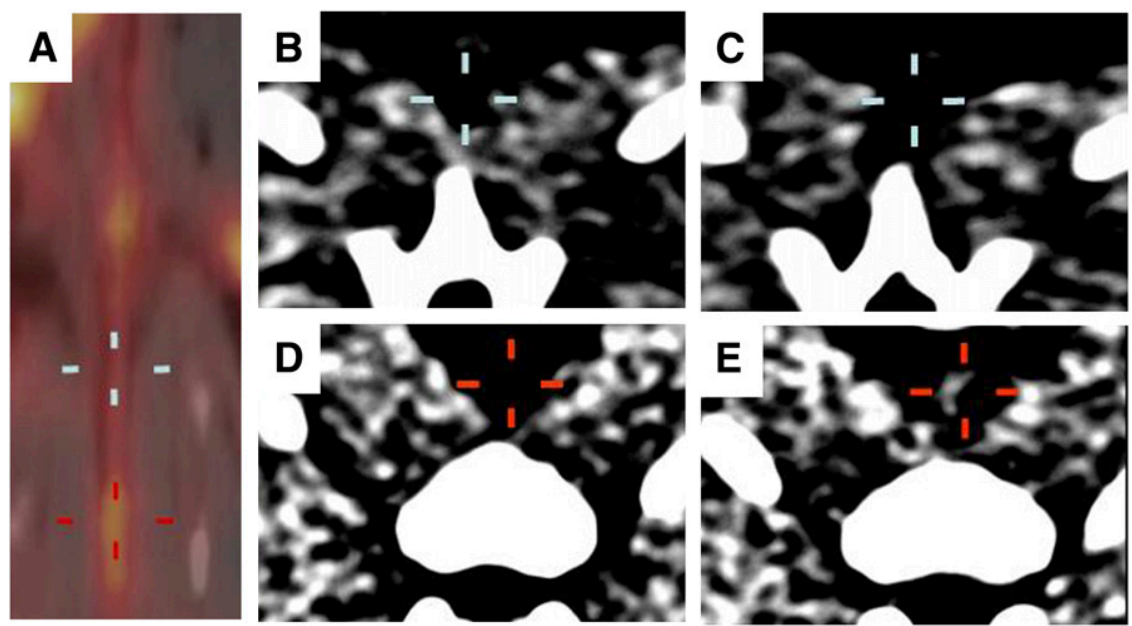

causing strong $\mathrm{T} 2 *$-shortening effects $(20,21)$ that can be detected as signal voids using MRI. In carotid atherosclerotic plaques $(22,23)$, focal signal voids can be detected on MRI after the injection of USPIO, localized to the
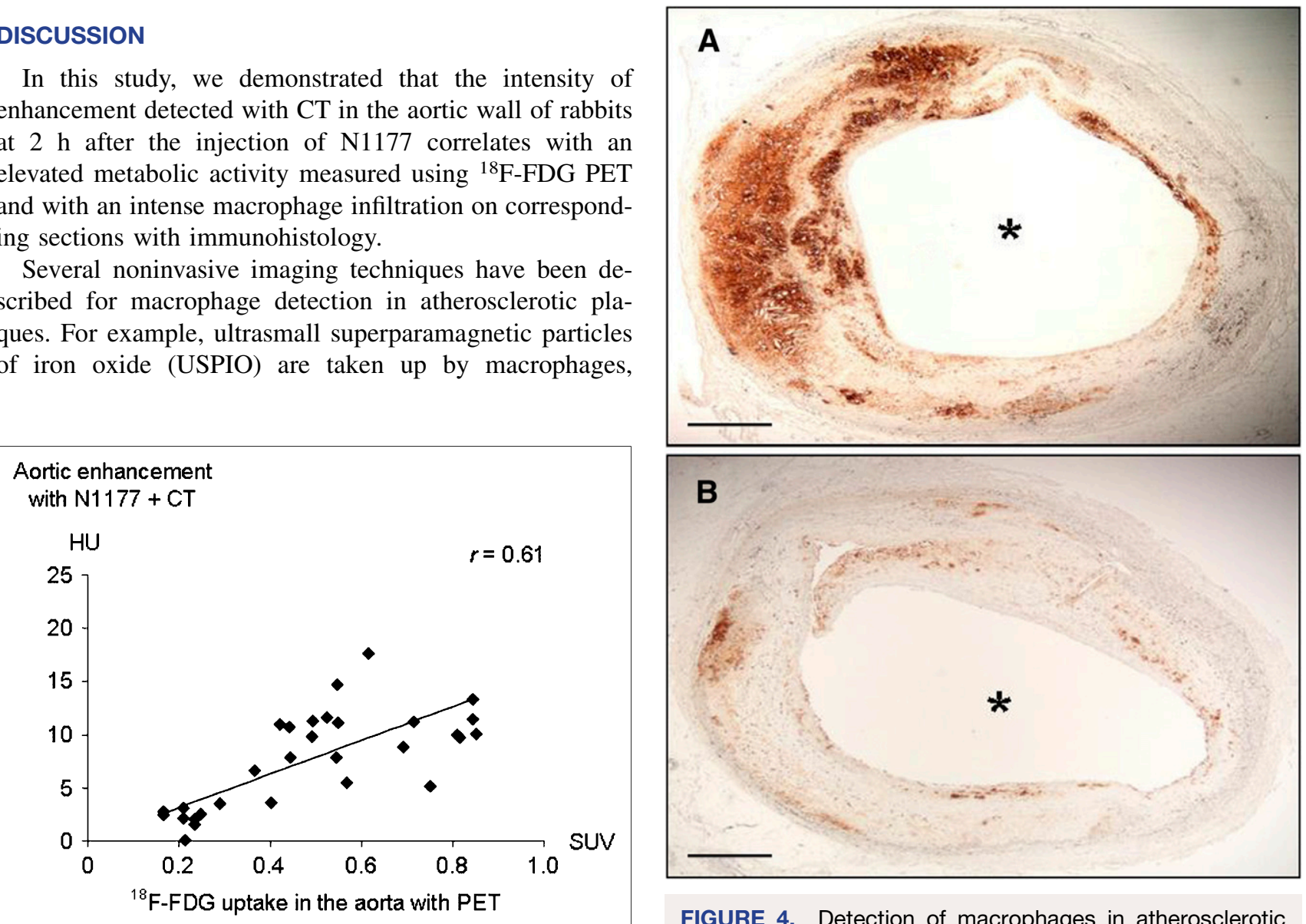

FIGURE 3. Correlation between N1177-enhanced CT and ${ }^{18}$ F-FDG PET. Correlation $(r=0.61)$ between intensity of enhancement measured $2 \mathrm{~h}$ after injection of N1177 with CT and SUVs detected at $3 \mathrm{~h}$ after the injection of ${ }^{18} \mathrm{~F}-\mathrm{FDG}$ with PET, in corresponding aortic segments from same rabbits.

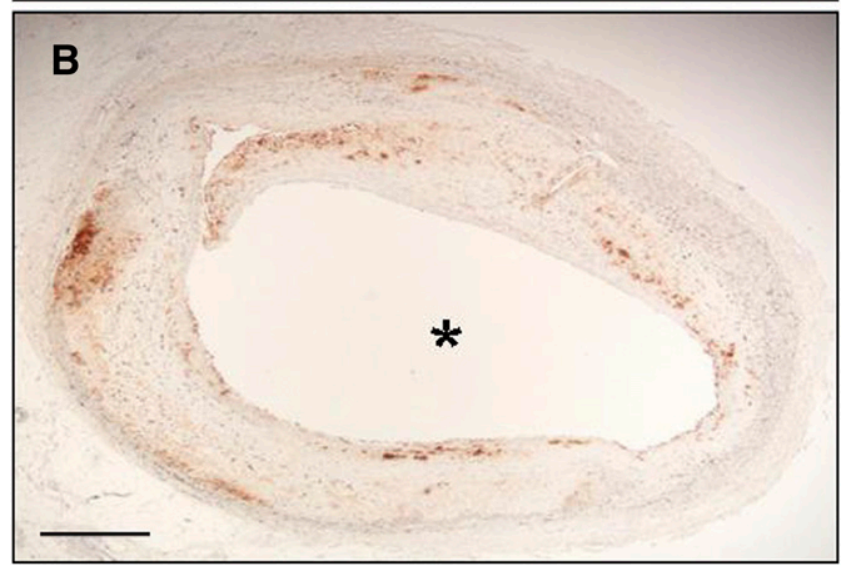

FIGURE 4. Detection of macrophages in atherosclerotic plaques by immunohistochemistry. Axial slices with intense and low enhancement detected with N1177 CT (Figs. 2E and $2 \mathrm{C}$, respectively) had strong $(A)$ and weak $(B)$ macrophage infiltration on corresponding axial cross-sections of atherosclerotic plaques by immunohistology. ${ }^{*}=$ lumen. Bar width $=$ $1 \mathrm{~mm}$. 


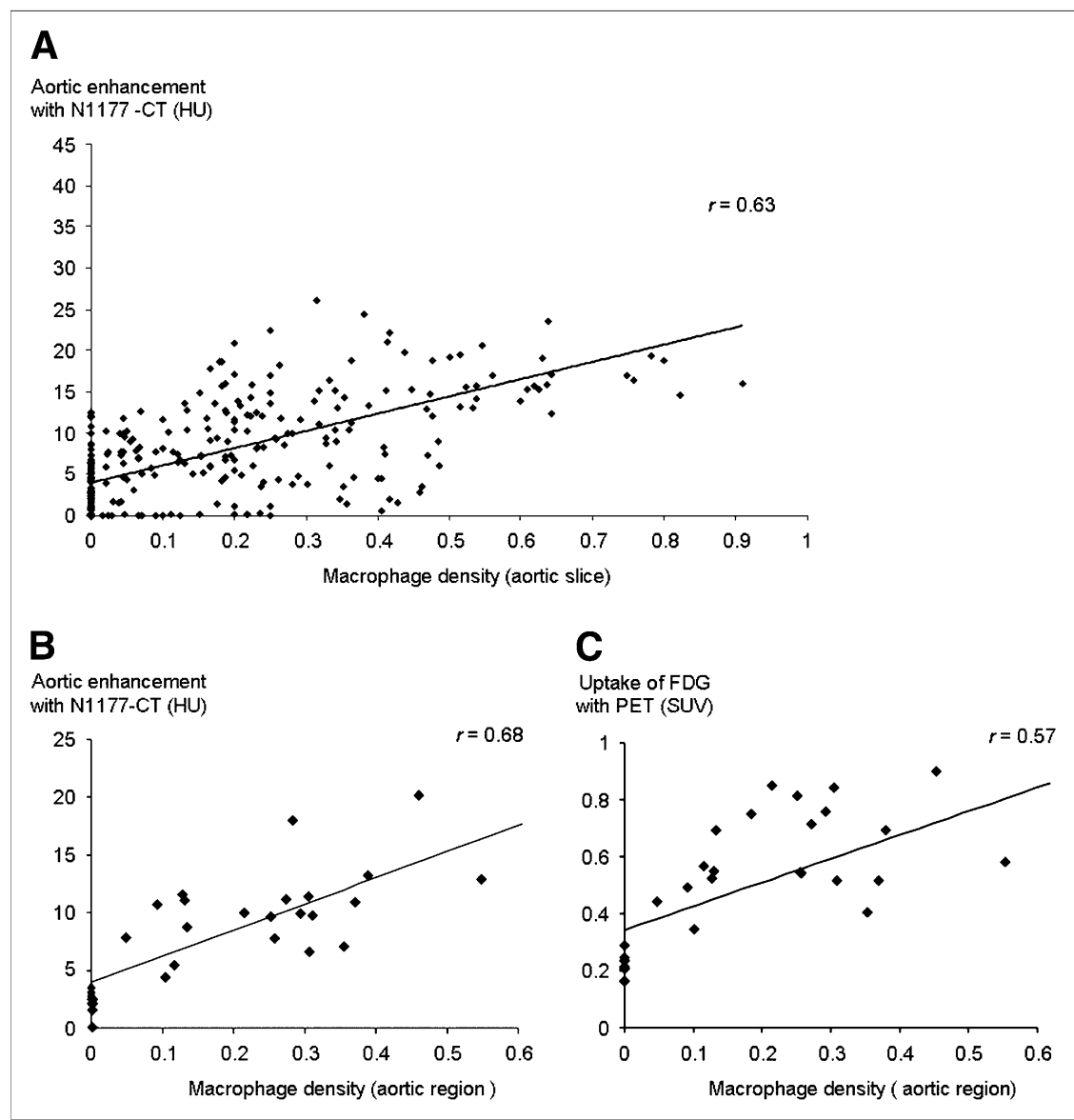

FIGURE 5. Correlation between N1177-enhanced CT and macrophage density. (A) Correlation between intensity of enhancement measured with CT $2 \mathrm{~h}$ after injection of N1177 and macrophage density on corresponding aortic cross-sections $(r=0.63)$. Correlations between macrophage densities measured in aortic regions and intensities of enhancement in HUs with N1177 CT (B) and SUVs with ${ }^{18}$ F-FDG PET (C).

macrophage-rich regions that accumulated iron particles on histology. However, precise quantification of USPIO accumulation in macrophages using MRI is limited by concomitant $\mathrm{T} 1$-shortening effects caused by nonspecific accumulation of USPIO in plaques (24) and T2*-effects related to plaque hemorrhage and calcifications (25). Additional inaccuracies may arise when attempts are made to distinguish the negative contrast due to USPIO accumulation from that secondary to vessel calcification or inherent image artifacts, although positive contrast methods such as gradient echo acquisition for superparamagnetic particles/ susceptibility may help the image interpretation (26). In recent work (27), a radiotracer was bound to iron oxide nanoparticles to quantify the accumulation of these particles using PET. Indeed, Nahrendorf et al. (27) demonstrated a strong correlation between the activity measured with PET and macrophage content in corresponding sections of atherosclerotic plaques. However, high spatial and temporal imaging resolutions required for imaging the arterial wall of coronary arteries are currently achievable neither with PET nor with MRI but are available with clinical MDCT. In addition, the optimal imaging time after the injection of iron oxide nanoparticles may be up to $72 \mathrm{~h}$ (23), which clearly limits the practical use of this technique as a screening tool.
In contrast, CT offers detection of macrophage-rich lesions as early as $2 \mathrm{~h}$ after the intravenous injection of N1177 with a spatial resolution that permits the evaluation of coronary atherosclerotic plaques. Quantification of N1177 accumulation in atherosclerotic plaques is facilitated by the linear relationship existing between iodine concentration in tissue and signal increase measured with CT. In addition, detection of macrophages with N1177 could be associated with other CT markers identified in ruptured plaques such as areas of low densities, positive remodeling, and absence of calcifications, enhancing the potential of CT to identify high-risk plaques $(28,29)$. N1177 CT will, however, need to overcome some important issues before clinical application to imaging inflammation in coronary atherosclerotic plaques is considered. First, additional studies are currently being performed to confirm the tolerability of N1177 at the dose used in this study. Second, changes in plaque density detected with N1177 CT in the aorta of atherosclerotic rabbits were small and could be difficult to detect in human coronary arteries subject to cardiac and respiratory motion. In fact, the technique used in this study to measure plaque enhancement with N1177 CT (averaging 12 ROIs adjacent to the aortic lumen) offered a good reproducibility of the measurements but clearly underestimated focal enhancement, which could 
reach up to $40 \mathrm{HUs}$ in some areas of atherosclerotic plaques. Third, whether high CT densities from accumulation of N1177 could be discriminated from those due to plaque calcifications in humans needs to be determined. The development of dual-energy $\mathrm{x}$-ray beam technology (30,31), based on simultaneous CT acquisitions using 2 beams at different energy levels, may help to discriminate between radiographic absorption properties of iodine and calcium in atherosclerotic plaques.

${ }^{18} \mathrm{~F}-\mathrm{FDG}$ enters into cells through glucose transporter proteins, which are overexpressed in cells with high metabolic activities. A previous study ( 8 ) demonstrated by ex vivo autoradiography that ${ }^{18} \mathrm{~F}-\mathrm{FDG}$ accumulated predominantly in macrophages in atherosclerotic plaques. Because activation of macrophages stimulates metabolic activity (32), ${ }^{18}$ F-FDG uptake measured in atherosclerotic plaques reflects both macrophage density and activity. In contrast, similar to iron oxide nanoparticles, N1177 accumulates in plaques through phagocytosis by macrophages (19). Interestingly, a strong correlation was observed between USPIO and ${ }^{18}$ F-FDG uptake in human carotid plaques imaged using MRI and PET, respectively (33). Therefore, the good correlation found in this study between $\mathrm{N} 1177$ and ${ }^{18} \mathrm{~F}-$ FDG accumulation in the aortic wall can be explained by similar macrophage densities but may also indicate that the intensity of phagocytosis and metabolic activity are closely associated in activated macrophages. Together, this study suggests that the intensity of enhancement measured with N1177 CT correlates both with macrophage density and with activity in atherosclerotic plaques. However, some discrepancies were also found in this study between signal measured on the aortic wall with N1177 CT and ${ }^{18}$ F-FDG PET and may be at least partly explained by accumulation of ${ }^{18} \mathrm{~F}-\mathrm{FDG}$ in cells other than macrophages present in atherosclerotic plaques such as endothelial cells (34) and lymphocytes (35).

This study has a few limitations. First, we compared the signal obtained in 0.4-mm-thick axial CT slices with macrophage density measured on $4-\mu \mathrm{m}$-thick sections by immunohistology. Therefore, to provide a second means of validation, the results of N1177-enhanced CT were compared with the activity measured in vivo in the same rabbits using ${ }^{18} \mathrm{~F}-\mathrm{FDG}$ PET, as a surrogate for macrophage infiltration. In addition, the use of a combined PET/CT system allowed for an optimal matching between both imaging modalities. A second limitation is that CT, compared with other techniques such as MRI, requires radiation. Exposure to radiations could be a concern for patient imaging, particularly in the case of repeated studies, or if CT is used for the screening for vulnerable plaque. However, future technologic developments in CT scanners could help to overcome these current limitations. The use of highly efficient flat-panel detectors (36), tube current modulation, and prospective cardiac gating may allow a decrease in radiation exposure. Finally, the results obtained in an experimental model of atherosclerosis need to be validated in human complex atherosclerotic plaques.

\section{CONCLUSION}

We demonstrated in this study that the intensity of enhancement detected in the aortic wall of rabbits using N1177-enhanced CT strongly correlated with a high metabolic activity evaluated using ${ }^{18}$ F-FDG PET, with an intense macrophage infiltration on corresponding sections with immunohistology. N1177-enhanced CT represents a promising imaging modality for the noninvasive detection of macrophages in coronary atherosclerotic plaques. The identification of macrophages could be an important adjunct to morphologic markers for the detection of high-risk atherosclerotic plaques using CT.

\section{ACKNOWLEDGMENTS}

We thank Ash Rafique for his assistance in obtaining the PET/CT scans. Partial support was provided by NIH/NHLBI R01 HL71021 and NIH/NHLBI HL78667. This work was also supported in part by grants from the Federation Française de Cardiologie, the British Heart Foundation, and the NIHR Cambridge Biomedical Research Centre.

\section{REFERENCES}

1. Virmani R, Burke AP, Farb A, Kolodgie FD. Pathology of the vulnerable plaque. J Am Coll Cardiol. 2006;47(8, suppl):C13-C18.

2. Libby P. Current concepts of the pathogenesis of the acute coronary syndromes. Circulation. 2001;104:365-372.

3. Topol EJ, Nissen SE. Our preoccupation with coronary luminology: the dissociation between clinical and angiographic findings in ischemic heart disease. Circulation. 1995;92:2333-2342.

4. Fuster V, Moreno PR, Fayad ZA, Corti R, Badimon JJ. Atherothrombosis and high-risk plaque: part I-evolving concepts. J Am Coll Cardiol. 2005;46:937954.

5. Libby P. Inflammation in atherosclerosis. Nature. 2002;420:868-874.

6. Mauriello A, Sangiorgi G, Fratoni S, et al. Diffuse and active inflammation occurs in both vulnerable and stable plaques of the entire coronary tree: a histopathologic study of patients dying of acute myocardial infarction. J Am Coll Cardiol. 2005;45:1585-1593.

7. Fuster V, Fayad ZA, Moreno PR, Poon M, Corti R, Badimon JJ. Atherothrombosis and high-risk plaque: part II-approaches by noninvasive computed tomographic/magnetic resonance imaging. J Am Coll Cardiol. 2005;46:12091218 .

8. Rudd JH, Warburton EA, Fryer TD, et al. Imaging atherosclerotic plaque inflammation with $\left[{ }^{18} \mathrm{~F}\right]$-fluorodeoxyglucose positron emission tomography. Circulation. 2002;105:2708-2711.

9. Tawakol A, Migrino RQ, Bashian GG, et al. In vivo ${ }^{18}$ F-fluorodeoxyglucose positron emission tomography imaging provides a noninvasive measure of carotid plaque inflammation in patients. J Am Coll Cardiol. 2006;48:18181824.

10. Ogawa M, Ishino S, Mukai T, et al. ${ }^{18} \mathrm{~F}-\mathrm{FDG}$ accumulation in atherosclerotic plaques: immunohistochemical and PET imaging study. J Nucl Med. 2004;45: 1245-1250.

11. Tawakol A, Migrino RQ, Hoffmann U, et al. Noninvasive in vivo measurement of vascular inflammation with F-18 fluorodeoxyglucose positron emission tomography. J Nucl Cardiol. 2005;12:294-301.

12. Zhang Z, Machac J, Helft G, et al. Non-invasive imaging of atherosclerotic plaque macrophage in a rabbit model with F-18 FDG PET: a histopathological correlation. BMC Nucl Med. 2006;6:3.

13. Davies JR, Rudd JH, Fryer TD, et al. Identification of culprit lesions after transient ischemic attack by combined ${ }^{18} \mathrm{~F}$ fluorodeoxyglucose positron-emission tomography and high-resolution magnetic resonance imaging. Stroke. 2005;36: 2642-2647.

14. Dunphy MP, Freiman A, Larson SM, Strauss HW. Association of vascular ${ }^{18}$ F-FDG uptake with vascular calcification. J Nucl Med. 2005;46:12781284 . 
15. Achenbach S, Moselewski F, Ropers D, et al. Detection of calcified and noncalcified coronary atherosclerotic plaque by contrast-enhanced, submillimeter multidetector spiral computed tomography: a segment-based comparison with intravascular ultrasound. Circulation. 2004;109:14-17.

16. Achenbach S, Ropers D, Hoffmann U, et al. Assessment of coronary remodeling in stenotic and nonstenotic coronary atherosclerotic lesions by multidetector spiral computed tomography. J Am Coll Cardiol. 2004;43:842-847.

17. Pohle K, Achenbach S, MacNeill B, et al. Characterization of non-calcified coronary atherosclerotic plaque by multi-detector row CT: comparison to IVUS. Atherosclerosis. 2007;190:174-180.

18. Leber AW, Knez A, von Ziegler F, et al. Quantification of obstructive and nonobstructive coronary lesions by 64-slice computed tomography: a comparative study with quantitative coronary angiography and intravascular ultrasound. J Am Coll Cardiol. 2005;46:147-154.

19. Hyafil F, Cornily JC, Feig JE, et al. Noninvasive detection of macrophages using a nanoparticulate contrast agent for computed tomography. Nat Med. 2007;13: 636-641.

20. Hyafil F, Laissy JP, Mazighi M, et al. Ferumoxtran-10-enhanced MRI of the hypercholesterolemic rabbit aorta: relationship between signal loss and macrophage infiltration. Arterioscler Thromb Vasc Biol. 2006;26:176-181.

21. Ruehm SG, Corot C, Vogt P, Kolb S, Debatin JF. Magnetic resonance imaging of atherosclerotic plaque with ultrasmall superparamagnetic particles of iron oxide in hyperlipidemic rabbits. Circulation. 2001;103:415-422.

22. Kooi ME, Cappendijk VC, Cleutjens KB, et al. Accumulation of ultrasmall superparamagnetic particles of iron oxide in human atherosclerotic plaques can be detected by in vivo magnetic resonance imaging. Circulation. 2003;107: 2453-2458.

23. Trivedi RA, U-King-Im JM, Graves MJ, et al. In vivo detection of macrophages in human carotid atheroma: temporal dependence of ultrasmall superparamagnetic particles of iron oxide-enhanced MRI. Stroke. 2004;35:1631-1635.

24. Trivedi RA, Mallawarachi C, U-King-Im JM, et al. Identifying inflamed carotid plaques using in vivo USPIO-enhanced MR imaging to label plaque macrophages. Arterioscler Thromb Vasc Biol. 2006;26:1601-1606.

25. Yuan C, Mitsumori LM, Ferguson MS, et al. In vivo accuracy of multispectral magnetic resonance imaging for identifying lipid-rich necrotic cores and intraplaque hemorrhage in advanced human carotid plaques. Circulation. 2001;104:2051-2056.

26. Briley-Saebo KC, Mulder WJ, Mani V, et al. Magnetic resonance imaging of vulnerable atherosclerotic plaques: current imaging strategies and molecular imaging probes. J Magn Reson Imaging. 2007;26:460-479.

27. Nahrendorf M, Zhang H, Hembrador S, et al. Nanoparticle PET-CT imaging of macrophages in inflammatory atherosclerosis. Circulation. 2008;117:379387.

28. Motoyama S, Kondo T, Sarai M, et al. Multislice computed tomographic characteristics of coronary lesions in acute coronary syndromes. J Am Coll Cardiol. 2007;50:319-326.

29. Leber A, von Ziegler F, Becker A, et al. Characteristics of coronary plaques before angiographic progression determined by multi-slice CT. Int J Cardiovasc Imaging. 2008;24:423-428.

30. Flohr TG, McCollough CH, Bruder H, et al. First performance evaluation of a dual-source CT (DSCT) system. Eur Radiol. 2006;16:256-268.

31. Boll DT, Hoffmann MH, Huber N, Bossert AS, Aschoff AJ, Fleiter TR. Spectral coronary multidetector computed tomography angiography: dual benefit by facilitating plaque characterization and enhancing lumen depiction. J Comput Assist Tomogr. 2006;30:804-811.

32. Deichen JT, Prante O, Gack M, Schmiedehausen K, Kuwert T. Uptake of $\left[{ }^{18} \mathrm{~F}\right]$ fluorodeoxyglucose in human monocyte-macrophages in vitro. Eur J Nucl Med Mol Imaging. 2003;30:267-273.

33. Tang TY, Moustafa RR, Howarth SP, et al. Combined PET-FDG and USPIOenhanced MR imaging in patients with symptomatic moderate carotid artery stenosis. Eur J Vasc Endovasc Surg. 2008;36:53-55.

34. Maschauer S, Prante O, Hoffmann M, Deichen JT, Kuwert T. Characterization of ${ }^{18} \mathrm{~F}-\mathrm{FDG}$ uptake in human endothelial cells in vitro. $\mathrm{J}$ Nucl Med. 2004;45:455460.

35. Ishimori T, Saga T, Mamede M, et al. Increased ${ }^{18} \mathrm{~F}-\mathrm{FDG}$ uptake in a model of inflammation: concanavalin A-mediated lymphocyte activation. J Nucl Med. 2002;43:658-663.

36. Gupta R, Grasruck M, Suess C, et al. Ultra-high resolution flat-panel volume CT: fundamental principles, design architecture, and system characterization. Eur Radiol. 2006;16:1191-1205. 